\title{
Ocorrência de Plantas Aquáticas nos Reservatórios da LIGHT-RJ ${ }^{1}$
}

\author{
Ocurrence of Aquatic Plants in the Light-RJ Reservoirs
}

\begin{abstract}
MARTINS, D. ${ }^{2}$, VELINI, E.D. ${ }^{2}$, PITELI, R.A. ${ }^{3}$, TOMAZELLA, M, S. ${ }^{4}$ e NEGRISOLI, E. ${ }^{5}$
RESUMO - O objetivo do presente trabalho foi caracterizar as comunidades infestantes de plantas aquáticas presentes nos reservatórios da Light-Sistema de Eletricidade S.A., localizada no município de Piraí-RJ. Os levantamentos foram realizados no período de julho a setembro de 1998. Os reservatórios analisados foram: Vigário, Pereira Passos e Lajes, sendo as quantidades de pontos amostrados de 19, 9 e 15, respectivamente. Em cada ponto amostrado fez-se a marcação das coordenadas geográficas e avaliou-se a porcentagem de ocupação do corpo d'água pelas espécies de plantas aquáticas presentes. Depois da identificação das plantas, pôde-se verificar quais eram as espécies mais freqüentes e a sua distribuição dentro do sistema de geração de energia. As espécies encontradas nos reservatórios foram: Brachiaria arrecta (Hack.) Stent.; Egeria densa Planch.; Eichhornia azurea (Sw.) Kunth.; Eichhornia crassipes (Mart.) Solms.; Hymenachne amplexicaulis (Rudge) Nees.; Panicum rivulare Trin.; Pistia stratiotis L.; Polygonum spp.; Sagitaria montevidensis Cham. \& Schlecht; Salvinia auriculata (Micheli) Adans; e Thypha dominguensis L.
\end{abstract}

Palavras-chave: planta aquática, levantamento, Light.

\begin{abstract}
This study aimed to describe the aquatic weeds infesting the Light-Electric System S.A. reservoirs, located in Pirai-RJ, Brazil. The survey was carried out from July to September 1998. The reservoirs analyzed were: Vigario, Pereira Passos and Lajes, with 19, 9 and 15 points being sampled, respectively. Sampled geographic coordinates were marked in each point and a visual estimate was made of the percentage of river water occupied by the aquatic plants. After plant identification, the most frequent species and their distribution were determined as follows: Brachiaria arrecta (Hack.) Stent.; Egeria densa Planch.; Eichhornia azurea (Sw.) Kunth.; Eichhornia crassipes (Mart.) Solms.; Hymenachne amplexicaulis (Rudge) Nees.; Panicum rivulare Trin.; Pistia stratiotis L.; Polygonum spp.; Sagitaria montevidensis Cham.\& Schlecht; Salvinia auriculata (Micheli) Adans and Thypha dominguensis $L$.
\end{abstract}

Key words: aquatic plant, survey, Light.

\section{INTRODUÇÃO}

O levantamento de infestação por plantas aquáticas faz-se necessário, pois permite verificar o grau de infestação das diferentes espécies e a distribuição destas nos corpos d'água estudados. A partir desses dados, decisões podem ser tomadas a respeito da maneira mais correta de interagir com essas plantas, desde um simples monitoramento de infestação e distribuição dessas espécies dentro de um sistema aquático, até uma prática de controle.

Recebido para publicação em 14.8.2002 e na forma revisada em 16.1.2003.

2 Prof Dr., Departamento de Produção Vegetal da Faculdade de Ciências Agronômicas - FCA-UNESP, Caixa Postal 237, 18603-970 Botucatu-SP, Brasil, <dago@ fca.unesp.br>. ${ }^{3}$ Prof. Dr., Departamento de Biologia Aplicada à Agropecuária - FCAVUNESP, Jaboticabal-SP. ${ }^{4}$ Eng.-Agr., Dr., Comitê de Defesa Sanitária Vegetal, SAA, Av. Brasil, 2340, 13073-001 Campinas-SP. ${ }_{5}^{5}$ Eng.-Agr. M.S., aluno de Pós-Graduação em Agricultura, nível de Doutorado, Departamento de Produção Vegetal - FCAUNESP, Botucatu-SP. 
As plantas aquáticas podem ser encontradas vegetando as margens de rios e reservatórios ou dentro dos mais diversos ambientes aquáticos, empregando diferentes mecanismos de adaptação para sobrevivência e desenvolvimento. Enquanto algumas espécies apresentam-se enraizadas em corpos d'água com fortes correntezas, outras somente podem viver em águas paradas ou estagnadas (Martins et al., 2002).

As plantas aquáticas que proliferam de forma desorganizada nos reservatórios e rios podem provocar vários problemas, como: acúmulo de lixo e outros sedimentos, proliferação de vetores de doenças, dificuldades na navegação e prejuízos ao turismo e à pesca; nestes casos, podem ser denominadas de daninhas. Estas plantas afetam o transporte de cargas das hidrovias, dificultando as rotas de navegação, além dos prejuízos às usinas hidrelétricas na geração de energia, o que incrementa os custos de manutenção (Marcondes \& Tanaka, 1997).

Apesar de ter inegável importância, o levantamento da ocorrência de plantas aquáticas ainda é um grande desafio, pois elas são de grupos bastante distintos (emersas, flutuantes, submersas) e ocorrem em ampla variação de ambientes (de áreas de várzeas até profundidades superiores a 10 metros), além de haver enormes variações de espécies e distribuição espacial de um ano para outro. Por esse motivo, há grande quantidade de metodologias de avaliação de infestações de plantas aquáticas, podendo ser atividades simples, envolvendo apenas a identificação das espécies em campo, até o uso de imagens de satélite ou fotos aéreas, com quantificação das áreas ocupadas por cada espécie ou grupo (Malthus \& George, 1997; Jensen \& Davis, 1987).

Portanto, este projeto teve por objetivo levantar a ocorrência de plantas aquáticas presentes nos reservatórios da Light-Sistema de Eletricidade S.A., localizada no município de Piraí-RJ, por meio de incursões de barcos.

\section{MATERIAL E MÉTODOS}

Para melhor caracterizar as plantas presentes nos reservatórios, as espécies foram classificadas em dois grupos: plantas aquáticas emersas e imersas.
Durante o período de coleta dos dados, de julho a setembro de 1998, percorreu-se com barcos as margens dos reservatórios de Vigário, Pereira Passos e Lajes, sendo as quantidades de pontos amostrados de 19, 9 e 15, respectivamente. As áreas com ocorrência de plantas aquáticas foram fotografadas e identificadas com um aparelho localizador geográfico Garmin GPS 12.

Neste levantamento preliminar, a demarcação dos pontos de amostragens foi determinada a cada cinco minutos de navegação, em que se fazia a leitura das coordenadas no localizador geográfico e, com o auxílio de um gancho, arrastava-se no fundo do reservatório para verificar a presença ou ausência da plantas submersas. Nos pontos com plantas emersas e submersas atingindo a superfície, fez-se a identificação das espécies e a avaliação da área total infestada e da composição da participação de cada espécie (em porcentagem da área total). Nos pontos em que as plantas submersas não atingiam a superfície da água, não se fez a avaliação da área infestada, sendo registrada apenas a presença da espécie. Nos casos em que não era possível identificar a espécie, uma amostra foi coletada e preservada em sacos plásticos com água, para posterior identificação em laboratório.

\section{RESULTADOS}

Na Tabela 1 estão apresentadas as espécies de plantas aquáticas encontradas nos levantamentos dos reservatórios, onde foram identificadas e classificadas (nome científico, família, nome vulgar, código e grupo). Observou-se a ocorrência de 9 famílias e de 11 espécies de plantas aquáticas.

Na Tabela 2 estão apresentadas as espécies de plantas daninhas aquáticas, emersas e imersas, que ocorreram no reservatório de Vigário por ocasião do levantamento realizado em julho de 1998, bem como as coordenadas geográficas dos pontos de avaliação e a porcentagem de cobertura proporcionada pela flora infestante.

Eichhornia azurea constitui-se na principal espécie daninha ( 15 pontos de ocorrência), com $24,54 \%$ de cobertura da área amostrada em média, seguida de Salvinia auriculata (16 pontos de ocorrência), com $20,41 \%$ de 
cobertura da área amostrada em média, e de Brachiaria arrecta (8 pontos de amostragem), com 19,29\% de cobertura da área amostrada em média. Nota-se, ainda, a importância do Panicum rivulare.

Na Tabela 3 apresentam-se as espécies de plantas daninhas aquáticas, emersas e imersas, que ocorreram no reservatório de Pereira Passos, assim como as coordenadas geográficas dos pontos de avaliação e a porcentagem de cobertura proporcionada pela flora infestante. Nota-se que, neste reservatório, a incidência de plantas daninhas aquáticas foi muito baixa e a variabilidade de espécies

Tabela 1 - Espécies de plantas aquáticas identificadas nos reservatórios da Light-Piraí-RJ

\begin{tabular}{|l|l|l|c|c|}
\hline \multicolumn{1}{|c|}{ Nome científico } & \multicolumn{1}{|c|}{ Família } & \multicolumn{1}{|c|}{ Nome vulgar } & Código & Grupo \\
\hline Brachiaria arrecta (Hack.) Stent. & Poaceae & tanner-grass & BRASU & EM \\
\hline Egeria densa Planch. & Hydrocharitaceae & egéria, elódia-brasilienses & EGRDE & SU \\
\hline Eichhornia azurea (Sw.) Kunth. & Pontederiaceae & aguapé-de-cordão & EICAZ & EM \\
\hline Eichhornia crassipes (Mart.) Solms. & Pontederiaceae & aguapé-de-flor-roxa & EICCR & FL \\
\hline Hymenachne amplexicaulis (Rudge) Neees. & Poaceae & capim-capivara & HYVAN & AN \\
\hline Panicum rivulare Trin. & Poaceae & capim-santa-fé & PANRV & AN \\
\hline Pistia stratiotis L. & Araceae & alface-d'água & PISST & FL \\
\hline Polygonum spp.* & Polygonaceae & erva-de-bico & POL & EM \\
\hline Sagitaria montevidensis Cham. \& Schlecht & Alismataceae & aguapé-de-flexa & SAGMO & EF \\
\hline Salvinia auriculata (Micheli) Adans & Salvinaceae & erva-de-sapo & SAVAV & FL \\
\hline Thypha dominguensis L. & Typhaceae & taboa & TYPLA & EM \\
\hline
\end{tabular}

* Polygunum lapathipholium e Polygunum ferrugineum.

Grupo EM - emersa; FF - emersa com folhas flutuantes; FL - flutuantes; SU - submersa; NA - ancorada na margem.

Tabela 2 - Espécies de plantas daninhas presentes no reservatório de Vigário. Coordenadas dos pontos de avaliação e porcentagem de cobertura de superfície do lago pelas espécies imersas e emersas presentes. Pontos de 1 a 10

\begin{tabular}{|c|c|c|c|c|c|c|c|c|c|c|}
\hline \multirow{3}{*}{$\begin{array}{r}\text { Latitude } \\
\text { Longitude }\end{array}$} & \multicolumn{10}{|c|}{ Ponto de Avaliação } \\
\hline & $22^{\circ} 40^{\prime} 57,4 "$ & $22^{\circ} 40^{\prime} 39,3^{\prime \prime}$ & $22^{\circ} 40^{\prime} 18,3^{\prime \prime}$ & $22^{\circ} 40^{\prime} 0,52^{\prime \prime}$ & $22^{\circ} 40^{\prime} 0,87^{\prime \prime}$ & $22^{\circ} 39^{\prime} 65,6^{\prime \prime}$ & $22^{\circ} 39^{\prime} 40,2^{\prime \prime}$ & $22^{\circ} 39^{\prime} 12,1^{\prime \prime}$ & $22^{\circ} 38^{\prime} 97,7^{\prime \prime}$ & $22^{\circ} 38^{\prime} 63,7^{\prime \prime}$ \\
\hline & $43^{\circ} 53^{\prime} 0,18^{\prime \prime}$ & $43^{\circ} 52^{\prime} 82,5^{\prime \prime}$ & $43^{\circ} 52^{\prime} 75,2^{\prime \prime}$ & $43^{\circ} 52^{\prime} 77,0^{\prime \prime}$ & $43^{\circ} 53^{\prime} 0,68^{\prime \prime}$ & $43^{\circ} 53^{\prime} 13,5^{\prime \prime}$ & $43^{\circ} 53 \prime 22,8^{\prime \prime}$ & $43^{\circ} 53^{\prime} 21,5^{\prime \prime}$ & $43^{\circ} 52^{\prime} 85,8^{\prime \prime}$ & $43^{\circ} 52^{\prime} 85,8^{\prime \prime}$ \\
\hline Espécie & 1 & 2 & 3 & 4 & 5 & 6 & 7 & 8 & 9 & 10 \\
\hline Brachiaria arrecta & 0,00 & 0,00 & 2,00 & 0,00 & 0,00 & 0,10 & 0,00 & 0,00 & 0,00 & 95,00 \\
\hline Eichhornia azurea & 1,00 & 0,00 & 4,00 & 0,10 & 25,00 & 0,10 & 55,00 & 65,00 & 80,00 & 0,40 \\
\hline Eichhornia crassipes & 0,60 & 0,00 & 0,10 & 0,00 & 0,00 & 0,00 & 0,00 & 1,00 & 0,00 & 0,00 \\
\hline Hymenachne amplexicaulis & 0,00 & 0,00 & 0,00 & 0,00 & 0,00 & 0,00 & 0,00 & 0,00 & 0,00 & 0,00 \\
\hline Panicum rivulare & 0,00 & 0,00 & 0,00 & 0,00 & 34,00 & 0,00 & 0,00 & 0,00 & 0,00 & 0,30 \\
\hline Pistia stratiotis & 4,00 & 1,00 & 0,50 & 0,10 & 8,00 & 0,10 & 2,00 & 6,00 & 3,00 & 0,30 \\
\hline Sagitaria montevidensis & 0,00 & 1,00 & 0,00 & 0,00 & 0,00 & 0,00 & 0,00 & 0,00 & 0,00 & 0,00 \\
\hline Salvinia auriculata & 94,00 & 0,00 & 90,00 & 0,10 & 30,00 & 0,10 & 43,00 & 25,00 & 17,00 & 4,00 \\
\hline
\end{tabular}

Tabela 2, cont. - Pontos de 11 a 19

\begin{tabular}{|c|c|c|c|c|c|c|c|c|c|c|}
\hline \multirow{3}{*}{$\begin{array}{l}\text { Latitude } \\
\text { Longitude }\end{array}$} & \multicolumn{10}{|c|}{ Ponto de Avaliação } \\
\hline & $22^{\circ} 38^{\prime} 44,6^{\prime \prime}$ & $22^{\circ} 38 ' 50,6^{\prime \prime}$ & $22^{\circ} 37^{\prime} 12,3^{\prime \prime}$ & $22^{\circ} 37^{\prime} 93,1^{\prime \prime}$ & $22^{\circ} 38^{\prime} 44,6^{\prime \prime}$ & $22^{\circ} 38^{\prime} 74,8^{\prime \prime}$ & $22^{\circ} 38^{\prime} 92,7^{\prime \prime}$ & $22^{\circ} 39^{\prime} 68,3^{\prime \prime}$ & $22^{\circ} 40^{\prime} 20,4^{\prime \prime}$ & \\
\hline & $43^{\circ} 52^{\prime} 12,3^{\prime \prime}$ & $43^{\circ} 52^{\prime} 45,6^{\prime \prime}$ & $43^{\circ} 53^{\prime} 78,0^{\prime \prime}$ & $43^{\circ} 53^{\prime} 67,9^{\prime \prime}$ & $43^{\circ} 53 ' 33,7^{\prime \prime}$ & $43^{\circ} 53^{\prime} 32,2^{\prime \prime}$ & $43^{\circ} 53^{\prime} 0,85^{\prime \prime}$ & $43^{\circ} 52^{\prime} 99,3^{\prime \prime}$ & $43^{\circ} 52^{\prime} 88,6^{\prime \prime}$ & \\
\hline Espécie & 11 & 12 & 13 & 14 & 15 & 16 & 17 & 18 & 19 & Total \\
\hline Brachiaria arrecta & 70,00 & 2,00 & 0,00 & 0,00 & 0,00 & 5,00 & 0,00 & 94,50 & 0,00 & 19,29 \\
\hline Eichhornia azurea & 0,50 & 55,00 & 0,00 & 0,10 & 70,00 & 60,00 & 50,00 & 0,00 & 0,10 & 24,54 \\
\hline Eichhornia crassips & 0,50 & 0,00 & 0,00 & 0,00 & 0,00 & 1,00 & 0,00 & 0,00 & 0,00 & 0,17 \\
\hline Panicum rivulare & 0,00 & 0,00 & 99,00 & 0,00 & 25,00 & 0,00 & 0,10 & 1,50 & 0,00 & 8,42 \\
\hline Pistia stratiotis & 2,00 & 0,00 & 0,00 & 0,10 & 1,00 & 30,00 & 9,50 & 0,10 & 0,10 & 3,56 \\
\hline Salvinia auriculata & 27,00 & 8,00 & 0,00 & 0,10 & 4,00 & 2,00 & 40,00 & 3,50 & 0,10 & 20,41 \\
\hline
\end{tabular}


Tabela 3 - Espécies de plantas daninhas presentes no reservatório de Pereira Passos. Coordenadas dos pontos de avaliação e porcentagem de cobertura de superfície do lago pelas espécies imersas e emersas presentes. Pontos de 1 a 9

\begin{tabular}{|c|c|c|c|c|c|c|c|c|c|c|}
\hline \multirow{3}{*}{$\begin{array}{r}\text { Latitude } \\
\text { Longitude }\end{array}$} & \multicolumn{10}{|c|}{ Ponto de Avaliação } \\
\hline & $22^{\circ} 41^{\prime} 12,1^{\prime \prime}$ & $22^{\circ} 41^{\prime} 10,6^{\prime \prime}$ & $22^{\circ} 41^{\prime} 02,5^{\prime \prime}$ & $22^{\circ} 41^{\prime} 01,7^{\prime \prime}$ & $22^{\circ} 41^{\prime} 01,7^{\prime \prime}$ & $22^{\circ} 41^{\prime} 12,5^{\prime \prime}$ & $22^{\circ} 41^{\prime} 27,6^{\prime \prime}$ & $22^{\circ} 41^{\prime} 31,3^{\prime \prime}$ & $22^{\circ} 41^{\prime} 33,0^{\prime \prime}$ & \\
\hline & $43^{\circ} 49^{\prime} 29,5^{\prime \prime}$ & $43^{\circ} 49^{\prime} 44,2^{\prime \prime}$ & $43^{\circ} 49^{\prime} 45,0^{\prime \prime}$ & $43^{\circ} 50^{\prime} 01,5^{\prime \prime}$ & $43^{\circ} 49^{\prime} 56,7^{\prime \prime}$ & $43^{\circ} 50^{\prime} 34,0^{\prime \prime}$ & $43^{\circ} 50^{\prime} 53,7^{\prime \prime}$ & $43^{\circ} 51^{\prime} 37,3^{\prime \prime}$ & $43^{\circ} 51^{\prime} 37,3^{\prime \prime}$ & \\
\hline Espécie & 1 & 2 & 3 & 4 & 5 & 6 & 7 & 8 & 9 & Total \\
\hline Brachiaria arrecta & 0,00 & 0,00 & 2,00 & 0,00 & 0,00 & 0,00 & 0,00 & 0,00 & 0,00 & 0,20 \\
\hline Eichhornia azurea & 0,00 & 0,00 & 4,00 & 0,10 & 0,00 & 0,00 & 0,00 & 0,00 & 0,00 & 0,50 \\
\hline Eichhornia crassipes & 0,00 & 0,00 & 0,10 & 0,00 & 0,00 & 0,00 & 0,00 & 0,00 & 0,00 & 0,00 \\
\hline Pistia stratiotis & 0,00 & 0,00 & 0,50 & 0,10 & 0,00 & 0,00 & 0,00 & 0,00 & 0,00 & 0,10 \\
\hline Salvinia auriculata & 0,00 & "' 0,00 & 88,00 & 0,10 & 0,00 & 0,00 & 0,00 & 0,00 & 0,00 & 9,80 \\
\hline
\end{tabular}

foi pequena, ocorrendo apenas cinco espécies em apenas dois pontos de amostragem.

Não foi observada infestação de plantas daninhas aquáticas ( 15 pontos) no reservatório de Lajes.

\section{LITERATURA CITADA}

JENSEN, J. R.; DAVIS, B. A. Remote sensing of aquatic macrophyte distribution in selected South Carolina reservoirs. In: ASPRS-ACSM ANNUAL CONVENTION: Remote Sensing. Baltimore. Proceedings... Fall Church: ASPRS-ACSM, 1987. v. 1. p.57-65.
MALTHUS, T. J.; GEORGE, D. G. Airborne remote sensing of macrophytes in Cefni Reservoir, Anglesey, UK. Aquatic Bot., v. 58, p. 317-332, 1997.

MARCONDES, D. A. S.; TANAKA, R. H. Plantas aquáticas nos reservatórios das usinas hidrelétricas da CESP. In: CONGRESSO BRASILEIRO DA CIÊNCIA DAS PLANTAS DANINHAS, 21., WORKSHORP DE PLANTAS AQUÁTICAS, 1997, Caxambu. Anais... Caxambu: SBCPD, 1997. p. 2-4.

MARTINS, D. et al. Controle químico de Pistia stratiotes, Eichhornia crassipes e Salvínia molesta em caixas d'água. Planta Daninha, v. 20, p. 83-89, 2002. (Edição Especial). 